\title{
The welding process impact on residual stress and distortion
}

Paul Colegrove ${ }^{1}$, Chukwugozie Ikeagu ${ }^{2}$, Adam Thistlethwaite ${ }^{3}$, Stewart Williams ${ }^{1}$, Tamas Nagy ${ }^{1}$, Wojciech Suder ${ }^{1}$, Axel Steuwer ${ }^{4}$ and Thilo Pirling ${ }^{5}$.

${ }^{1}$ Welding Engineering Research Centre, Cranfield University, Bedfordshire MK43 0AL, UK

${ }^{2}$ Acergy, Bucksburn House, Howes Road, Aberdeen, AB16 7QU, UK

${ }^{3}$ Furmanite International Ltd - UK, Furman House, Shap Road, Kendal, Cumbria LA9 6RU, UK

${ }^{4}$ ESS Scandinavia, Lund University, Stora Algatan 4, 22350 Lund, Sweden

${ }^{5}$ Institut Laue-Langevin (ILL), BP 156, 6, rue Jules Horowitz, 38042 Grenoble Cedex 9, France 


\begin{abstract}
Residual stress and distortion continue to be important issues in shipbuilding and are still subject to large amounts of research. This paper demonstrates how the type of welding process influences the amount of distortion. Many shipyards currently use submerged arc welding (SAW) as their welding process of choice. In this manuscript we compare welds made by SAW with DC gas metal arc welding, pulsed gas metal arc welding, Fronius Cold Metal Transfer (CMT), autogenous laser and laser hybrid welding on butt welds in $4 \mathrm{~mm}$ thick DH36 ship plate. Laser and laser hybrid welding were found to produce the lowest distortion. Nevertheless a considerable improvement can be achieved with the pulsed gas metal arc welding and CMT processes. The paper seeks to understand the relationship between heat input, fusion area, measured distortion and the residual stress predicted from a simple numerical model, and the residual stresses validated with experimental data.
\end{abstract}

Keywords: Welding, modelling, residual stress, distortion. 


\section{Introduction}

Two of the major problems of any welding process are residual stress and distortion. Extensive reviews of these problems have been written by Masubuchi ${ }^{1}$ and Radaj $^{2}$ and a more recent review which focuses on the issues in the shipbuilding industry has been written by $\mathrm{McPherson}^{3}$. Residual stress is primarily caused by the compressive yielding that occurs around the molten zone as the material heats and expands during welding. When the weld metal cools it contracts which causes a tensile residual stress, particularly in the longitudinal direction. These stresses are shown diagrammatically in Fig. 1(a). After welding a residual tensile stress remains across the weld centerline and causes a balancing compressive stress further from the weld zone as shown in Fig. 1(b). The tensile residual stress on the weld line reduces the fatigue strength and the toughness, particularly when combined with any notches or defects associated with the weld bead.

To relieve some of the residual stresses caused by the welding process, the structure deforms, causing distortion. There are several modes of distortion, but the one that is most common, particularly in thin welded structures is buckling distortion, which is caused by the compressive stress in the parent material. Buckling distortion is becoming increasingly common due to the use of new high strength materials that enable the use of thinner sections with reduced critical buckling loads (and stresses) ${ }^{4}$.

It is often useful to examine the residual stress in terms of the Applied Weld Load (AWL). The AWL is the magnitude of the longitudinal tensile load and is obtained by multiplying the average tensile stress $\sigma_{\max }$ by the width of the tensile stress region ${ }^{4}$. Where the AWL exceeds the critical buckling load distortion occurs ${ }^{5}$. To reduce the AWL it is necessary to reduce either the magnitude or the width of the tensile stress region.

Reducing the AWL can be done by either processing or stress engineering. The processing method involves the use of a lower heat input e.g. laser welding ${ }^{6,7}$ to reduce the width of the tensile stress region during welding. These methods generally have little effect on the magnitude of the tensile stress region which is typically around the yield value. Stress engineering methods are those that can be used during an existing welding process to lower the magnitude of the tensile stress region. These methods rely on the application of a tensile stress which reduces the compressive yielding and induces a tensile stretch in the material after welding ${ }^{8}$.

Finally, some methods reduce the distortion without significantly reducing the weld residual stress or AWL. These include improved clamping and weld sequencing ${ }^{9}$ and those methods that deal with the residual stress after it has been created. The most common methods are post weld heat treatment, flame straightening ${ }^{10}$, vibratory stress relief ${ }^{10}$ and induction heat treatment methods ${ }^{3}$.

This paper investigates the method of reducing the heat input to reduce the weld distortion and is an extension of the work reported in Nagy et al. ${ }^{11}$. It is divided into three parts, the first describes the experimental work, the second seeks to understand the experiments with a simple model that predicts the residual stress, and the final section compares these residual stress predictions with residual stress measurements from neutron diffraction. 


\section{Method}

\section{Welding}

The investigation compared six processes: Submerged Arc Welding (SAW), DC Gas Metal Arc Welding (GMAW), Pulsed GMAW, Fronius Cold Metal Transfer (CMT), autogenous laser and hybrid laser. The material used for the investigation was $500 \times 125 \times 4 \mathrm{~mm}$ ASTM A131 steel; grade DH36. The narrow width of the specimens ensured that the critical buckling load was low, so the distortion was large and could be easily measured. All the plates were rolled prior to welding to remove distortion (but not stress). Table 1 gives the typical compositional range. The yield strength is typically $350 \mathrm{MPa}$ and the ultimate tensile strength is between 490 and $620 \mathrm{MPa}$. All processes except for autogenous laser and SAW used ARCWELD A18 Plus, $1.2 \mathrm{~mm}$ diameter filler wire and a shielding gas that contained Argon with $20 \% \mathrm{CO}_{2}$. The SAW process used ESAB OK Autrod 12.22 copper coated, 3.2 mm diameter wire with Oerilikon OP 122 as the top flux and Oerilikon PIE UP 18 as the bottom flux. A closed square butt joint preparation and copper backing was used for all processes except CMT. This joint preparation was used because it requires the least preparation in a production environment. CMT used a single sided V-butt joint preparation with a $90^{\circ}$ included angle and a copper backing bar since the process had insufficient power to use the closed square butt preparation.

The clamping arrangement used for the experiments is shown in Fig. 2. The specimens were clamped to a large baseplate which had grooves for the backing bar and thermocouples. Consistent pressure along the weld length was crucial to producing high quality welds, due to its effect on the heat loss through the backing bar. Tacking of the plates was required to avoid plate separation during welding.

The thermocouples were spot welded to the underside of the plates due to the restrictions imposed by the clamps and the flux in SAW. Temperature was measured at distances of 15 , 30 and $60 \mathrm{~mm}$ from the weld centerline and K-type thermocouples were used at all locations. Monitoring of the current, voltage and wire feed speed was done with a Triton Electronics Limited's AMV 4000 arc watch system. This system calculated the average weld power input by averaging the instantaneous voltage multiplied by the instantaneous current. The weld heat input was then calculated by dividing by the weld travel speed and including an efficiency factor for the process. $85 \%$ was used for SAW and $80 \%$ was used for the GMAW processes based on $\mathrm{Kou}^{12}$. An efficiency of $90 \%$ was assumed for the laser power in the two laser processes based on Fuerschbach ${ }^{13}$. This issue is discussed further in a later section.

The welding parameters were developed by trial and error and are summarized in Table 2 . The welds were principally evaluated by visual examination and with BS EN 25817:1992 (ISO 5817:1992) intermediate quality level $\mathrm{C}$ as a guide. It should be noted that proper procedure development should involve other examinations such as metallography and mechanical testing (hardness and Charpy test). In using this standard, the following were ensured.

- Good bead penetration

- Adequate capping and smooth bead appearance

- Absence of surface defects such as undercuts.

\section{Post-weld Analysis}

The distortion of each of the plates was measured after welding with six Mitutoyo IDU Digimatic displacement transducers across a grid where the measurement points were spaced $50 \mathrm{~mm}$ apart. The length of each of the specimens was reduced to $450 \mathrm{~mm}$ to enable consistent distortion measurements. Welds from each of the processes were sectioned, 
polished and then etched with Nital (2\% Nitric acid in ethyl alcohol) before examination under the microscope.

Hardness was measured with a Vickers micro hardness testing machine. For SAW, DC GMAW, Pulsed GMAW and CMT, the indentation points were made $1 \mathrm{~mm}$ apart across the section, while measurements were made every $20 \mu \mathrm{m}$ for the hybrid and autogenous laser processes.

\section{Results}

High quality welds were obtained for all processes except DC GMAW and autogenous laser. The DC GMAW process was very sensitive to the welding parameters. Incorrect settings led to either burn-through (too much heat) or lack of penetration (too little). High quality welds were however achieved with the pulsed GMAW process: the lower heat input avoiding the problem of burn-through while the peak current provided sufficient arc pressure to achieve full penetration. The autogenous laser weld had significant undercut, which would be resolved by the addition of filler wire. In all welds, but particularly those with the higher heat inputs consistent clamping along the weld length was crucial to the production of high quality welds.

A typical plot of the measured distortion for SAW is shown in Fig. 3, and shows first order buckling distortion. First order buckling was observed for all the welds apart from autogenous laser weld where the distortion was negligible. The SAW also demonstrates a small amount of angular distortion which is just discernable in Fig. 3. The angular distortion for all other welding processes was negligible.

The distortion index for each weld was found by averaging the distortion measurements across the plate, which is plotted against the heat input in Fig. 4 (a). The trend line shows a near linear relationship between the heat input and distortion index. The greatest deviation from this trend is for the DC GMAW process. There is an even a stronger correlation between the heat input and the fusion area as shown in Fig. 4(b). Further evidence of the difference in heat inputs between the processes is provided by the plot of the peak temperatures verses the distance from the weld centerline in Fig. 5(a). Finally the residual stress measurements in Fig. 5(b) illustrate how the tensile peak widens with increasing heat input. There is very little difference in the magnitude of the tensile peak between the different welding processes. The results for the autogenous laser process are perhaps slightly lower, and there was considerably greater noise in these values than for the other processes.

Each of the welds was sectioned and the microstructure examined. Even though there were significant differences in the weld microstructures there was remarkably little difference in the hardness values of the base material, HAZ and weld metal as shown in Fig. 5(c). The one exception is the autogenous laser process which had an unacceptably high hardness in the fusion zone.

\section{Discussion}

Making an adequate comparison of the different welding processes is difficult given the widely different travel speeds used. Nevertheless, the high heat input and distortion of the SAW is abundantly clear: both being almost twice as much as the other arc welding processes. Therefore there is a clear advantage in using an alternate welding process to lower distortion. Overall, a distortion reduction of $20-70 \%$ is realizable with the GMAW processes over the SAW process and $80-95 \%$ reduction is possible with the laser processes. 
The near linear relationship between the heat input and fusion area in Fig. 4 (b) is perhaps more fortuitous for these set of experiments than a general rule that can be applied to all welding processes. Nevertheless higher heat inputs do result in more material being melted and they provide further verification of the heat input calculations.

As mentioned in the introduction, the amount of distortion depends on the applied weld load (AWL). For all processes except autogenous laser, the tensile strength in the weld region is nearly constant. Therefore the AWL is linearly dependent on the width of the tensile stress region. The width will be approximately proportional to the area of the fusion zone. Therefore the linear increase in fusion zone area with heat input, implies that there will also be a linear increase in the AWL. This link between AWL and heat input has also been demonstrated by James et al. ${ }^{4}$.

The other main finding, that heat input and distortion have a near linear relationship (Fig. 4 (a)) is supported by the findings of Michaeleris et al. ${ }^{14}$ who showed a near linear increase in distortion with AWL (and hence heat input as discussed above) above the critical buckling load. Furthermore, the trend line suggests that the critical buckling load is not exceeded for heat inputs below about $0.12 \mathrm{~kJ} \mathrm{~mm}^{-1}$ based on the $x$ axis intercept for this plate geometry. To summarize, there is a near linear relationship between the AWL, fusion area, heat input and distortion index for the welds analysed in this study.

The results for the residual stress measurements indicate that the width of the tensile peak increases with heat input, as expected. Note however that the residual stress of the CMT and Pulsed GMAW processes are almost identical. It should be noted however that the amount of reinforcement with the CMT weld is considerably less, in part due to the edge preparation. Therefore the Applied Weld Load for this weld will be lower than for the Pulsed GMAW which explains the greater distortion with this weld.

The peak temperatures provide further verification of the heat input findings, with a similar order being observed. Note, that some of the low speed processes, e.g. CMT, can have relatively high temperatures in the far field ( $60 \mathrm{~mm}$ away from the centerline) because there is more time for the heat to diffuse away from the arc.

Finally all of the weld hardness levels are within the acceptable level of $350 \mathrm{HV}^{7}$, with the exception of autogenous laser. In this case, the low heat input and high welding speeds contributed to a rapid cooling rate which led to the formation of lower bainite, and possibly some martensite being present. A more detailed analysis of a similar autogenous laser weld in DH 36 steel $^{7}$ showed that the weld metal contained bainite and Widmanstatten ferrite.

In addition, the autogenous laser process requires very tight tolerances and cannot tolerate any gaps between the plates. This leads to very poor process robustness and quality. To capture the differences between the processes, a qualitative comparison has been attempted in Table 3. Overall, the hybrid laser and pulsed GMAW have the most advantages and the least disadvantages. The final choice will depend on whether the hybrid laser's high productivity and low distortion offsets the added cost and safety issues associated with this process.

\section{Modeling}

\section{Description of Model}

3-dimensional models for welding the thermal cycle and residual stress in welding are now in common use as a research tool for both academic and commercial purposes ${ }^{15-19}$. The models 
use a transient 3-dimensional thermal model which is coupled to an elastic-plastic model for calculating the stress and strain. The loading on the elastic-plastic model is calculated from the thermal expansion at each time step. During the last time step the plate is allowed to cool, giving the as-welded residual stress. Computational time is often an issue with these models due to the need to calculate both the temperature and stress at each time step. Although a steady state approach is trivial for the heat flow, such an analysis cannot be used easily for residual stresses. Various methods have been used to reduce the solution time and include:

- 2-dimensional thermal and plane strain elastic-plastic models ${ }^{5}$. These models use a mesh which is orientated in a plane transverse to the welding direction. Although some accuracy is lost with the 2-dimensional approximation, the residual stress results compare favourably with experiments. The stress field from the 2-dimensional model can be applied to a three dimensional elastic model using a thermal loading, which enables the prediction of distortion ${ }^{14}$.

- Analytical methods ${ }^{20}$. These use a thermal model to calculate the maximum temperatures and the weld bead. The temperature data is then used to calculate the transverse 'Thermal Contraction Strain' (TCS) via the size of the weld bead and the longitudinal 'Mismatched Thermal Strain' (MTS) via the magnitude of the maximum temperature. These strains are then applied as a thermal load to a 3-dimensional elastic model which predicts the distortion. This method is similar to the previous one and differs in the analytical rather than computational method for calculating the thermal loading which makes it more efficient.

The model presented in this paper uses the former approach. It is used to get a better understanding of the differences between the processes through prediction of the longitudinal residual stress and in particular the far field compressive stress that causes buckling distortion. Note that prediction of distortion from the residual stress has not been attempted.

Although there are many similarities with the Michaleris and DeBiccari model, the current model differs from it in one major respect. Rather than using a 2-dimensional model for both the thermal and structural aspects, a 3-dimensional steady state model is used for the thermal field. A series of temperature slices from this model are shown in Fig. 6. These twodimensional temperature slices (in the $y-z$ plane) are then transferred to the 2-dimensional elastic-plastic model. Note that in practice many more slices are taken, particularly around the weld pool - the diagram in Fig. 6 is purely illustrative. Any differences with Michaleris and DeBiccari are expected to be minor, and due to forward heat conduction that is permissible in the 3-dimensional thermal model, but not accounted for in the simpler 2dimensional thermal model.

For each slice a plane strain analysis is carried out to determine the thermal strain (and hence stress) imparted by the temperature field. This strain is used as the initial condition for the analysis of the subsequent slice and this allows the development of stress to be determined. The stress analysis is carried out against the direction of welding i.e. from the cool base metal, through the heat source, along the trailing thermal gradients and back to metal at room temperature. This ensures that the final residual stress plot has taken account of the prior temperature and stress/strain history. With this in mind it is clear that it is important to minimize the distance between each slice if accurate stress predictions are required as important phenomena occurring between slices may be missed. Therefore the density of the slices around the weld region is increased due to the large thermal gradients.

\section{Model Application}

A model of each of the welds was created with the Comsol Finite Element package. The thermal properties used for this model are identical to those used in Michaleris and DeBiccari ${ }^{5}$ 
who used the properties for mild steel. There is little difference in the thermal properties with alloy content for structural steels. These properties use an artificially high thermal conductivity for temperatures greater than $1500^{\circ} \mathrm{C}$, to capture the convective heat transfer in the weld pool. The latent heat of fusion was included in the model by artificially increasing the specific heat around the melting temperature. The size of the latent heat was tested and had virtually no effect on the magnitude of the final residual stress.

The heat source was represented with a conventional double ellipsoid heat source ${ }^{21}$ for all processes including the laser processes.

$$
\begin{array}{ll}
q_{f}(x, y, z)=\frac{6 \sqrt{3} r_{f} Q}{a b c_{f} \pi \sqrt{\pi}} \exp \left(-3 \frac{x^{2}}{c_{f}^{2}}-3 \frac{y^{2}}{b^{2}}-3 \frac{z^{2}}{a^{2}}\right) \quad x>0 \\
q_{b}(x, y, z)=\frac{6 \sqrt{3} r_{b} Q}{a b c_{b} \pi \sqrt{\pi}} \exp \left(-3 \frac{x^{2}}{c_{b}^{2}}-3 \frac{y^{2}}{b^{2}}-3 \frac{z^{2}}{a^{2}}\right) \quad x<0
\end{array}
$$

where:

$x, y, z, a, b, c_{f}$ and $c_{b}$ are defined in Fig. 7;

$Q$ is the input power, including a factor for efficiency;

$r_{\mathrm{b}}$ and $r_{\mathrm{f}}$ are factors for distributing the power between the front and back of the weld.

They must add to 2 and have been distributed as:

$r_{f}=\frac{2 c_{f}}{c_{f}+c_{b}}$ and $r_{b}=\frac{2 c_{b}}{c_{f}+c_{b}}$

The authors acknowledge that better representations such as a point and line heat source $^{22}$ could have been used for the laser process, but this method was used to simplify the model's implementation. The size parameters $(a$, and $c$ ) used in the double ellipsoid heat source are shown in Table 4 and equal the weld pool size. Note that the thickness parameter, $b$ is assumed to be the plate thickness of $4 \mathrm{~mm}$. This is possibly not ideal, as Goldak and Akhlaghi $^{23}$ suggest using values $10 \%$ smaller than those of the weld, however the difference is small and not expected to have a significant effect on the residual stresses predicted. Finally, the travel speeds used for each process are shown in Table 2.

Heat loss occurs primarily from the underside which is in contact with the backing bar. This is modelled with a convective heat transfer coefficient which becomes one of the two main unknown variables in the model. The other unknown variable is the weld efficiency (which affects the weld power input). A trial and error approach was used to determine the value of these parameters. The thermal profile at the locations used for the thermocouples was determined and compared with the experimental results. By adjusting these two parameters the thermal profiles were matched to within $\pm 15^{\circ} \mathrm{C}$.

One of the most crucial aspects of the structural model is the material strength. The model uses elastic perfectly plastic material properties and ignores the phase changes that occur in the real material. The yield strength was varied between the processes, with the value being inferred from the average weld metal hardness. ASTM A370 Tables $2 \& 3$ were used to approximate tensile strength from the weld hardness values, and a tensile/yield strength factor of the 1.58 was used to determine the approximate room temperature yield strength of the weld metal in each case. Table 5 shows the yield strengths obtained using this technique.

Temperature dependent yield strength values for the weld metal were inferred by offsetting the standard curve for the welding wire from Fig. 5 of Michaleris and DeBiccari ${ }^{5}$ by the difference between the standard value and that stated in

Table 5. 


\section{Results}

Unfortunately it was not possible to obtain results for the SAW process due to instability problems that were likely to be caused by the high heat input for this process. No problems were experienced for the remaining five processes.

The weld efficiency and heat transfer coefficient values that gave best agreement with the thermocouples are shown in Table 6. The efficiency of all processes apart from CMT was lower than those reported in $\mathrm{Kou}^{12}$. Fig. 8 illustrates the predicted longitudinal residual stress for each process. The residual stresses are generally near the yield values and are high and narrow for the laser process and lower and broader for the remaining processes.

To understand how the residual stress relates to a loading that causes distortion, the residual stress predictions were multiplied by the area over which the stress acts. This value is plotted against the heat input and compared with the distortion index Fig. 9.

\section{Discussion}

Some caution needs to be used in interpreting the values of weld efficiency and convective heat transfer coefficient in Table 6. The solutions shown were the best fit for the simple model used and there may be other solutions possible, particularly where more complex boundary conditions on the underside of the weld are used. For example, models that capture the relatively good heat transfer to the backing bar near the weld region would be more realistic and would lead to different heat transfer coefficients and weld efficiencies than those reported.

In all cases except the CMT process, the efficiency predicted by the model was $10-20 \%$ lower than the generic values provided in Kou ${ }^{12}$. More recent arc efficiency measurements by Joseph et al. gave values in the range 67 to $82 \%$ for GMAW and are closer to those reported in this work. Measuring the arc efficiency is difficult and the method described above is likely to be less accurate than using direct calorimetric measurements ${ }^{24}$. The other unknown parameter calibrated in the weld was the convective heat transfer coefficient. This showed a significant variation between the welds which may (in-part) be due to variation in the clamping conditions.

The predictions of the longitudinal residual stress profile are typical of this kind of model ${ }^{5}$ and show a flat compressive stress distribution with distance from the weld. The laser process has the greatest peak due to the harder microstructure. The peak value found for the remaining processes is similar, but widens with the greater heat input resulting in a greater weld load. There is a direct relationship between the weld load and the measured distortion as seen in Fig. 9. Based on these results the critical buckling load is likely to be between 20-25 $\mathrm{kN}$. The distortion of the laser welds and the autogenous laser weld in particular is likely to be inherent in the plate and/or angular distortion. A very similar relationship between the AWL and distortion index has been reported in Michaleris et al. ${ }^{5,14}$. The main difference with the published work is that the distortion index is measured experimentally, rather than being an output of a model.

\section{Residual Stress Measurements}

\section{Methodology}

The residual stresses of each of the samples were measured by neutron diffraction on the SALSA Engineering Beamline at the Institut Laue-Langevin in Grenoble, France during beam time allocation 1-01-56. Each of the specimens was cut down to $385 \mathrm{~mm}$ in length for the 
purpose of this experiment, and the strains were measured transverse to the weld direction half way along the length. The residual stresses were inferred from the strains in the longitudinal, transverse and normal directions assuming a biaxial stress system. The SALSA instrument used a wavelength of $1.694 \AA$ and the diffraction peak $\mathrm{Fe}-211$ at $2 \Theta=88.5^{\circ}$ was measured to obtain cuboidal gauge volumes. The primary and secondary slits were positioned approximately $40 \mathrm{~mm}$ away from the gauge volume. The gauge volume, as defined by the slit sizes was $1 \times 1 \times 0.9 \mathrm{~mm}^{3}$ for the longitudinal strains and $1.5 \times 1.5 \times 10 \mathrm{~mm}^{3}$ for the normal and transverse directions. $1 \mathrm{~mm}$ steps were used to capture the residual stresses in the weld region which was increased to $10 \mathrm{~mm}$ in the parent material, away from the weld. Each measurement point counted 1500 neutrons to ensure a good quality diffraction peak. Finally, the strain free lattice spacing was found from equations that assumed the stress normal to the plate surface was zero (biaxiality).

\section{Results and Discussion}

The longitudinal residual stresses across the six welds are shown in Fig. 10(a) and a comparison of the weld region with the model predictions is shown in Fig. 10(b). The results indicate that the width of the tensile peak increases with heat input, as expected. Note however that the residual stress of the CMT and pulsed gas metal arc welding process are almost identical, indicating comparable (absorbed) heat input for the two processes.

The comparison between the model and experimental predictions in Fig. 10(b) is particularly interesting. Although the magnitude of the peak stresses is not well represented by the model, there is remarkably good agreement in the width of the tensile stress regime. This result is pleasing given the simplifying assumptions made in the model. The magnitude of the tensile peak produced by the model is primarily a function of the yield tensile stress vs. temperature data. Since this was not measured for the material used in the experiment it is hardly surprising that there is a discrepancy. In addition, there is very little variation in the peak residual stress between the processes, unlike the model predictions. In particular, the measured residual stress for the laser weld is slightly lower than the other processes and does not appear to be affected by microstructural hardening. It is unclear why this should be the case, although there was relatively large point-to-point scatter in the residual stress data for the laser weld.

\section{Conclusions}

Through a comparison of the SAW, DC GMAW, pulsed GMAW, CMT, hybrid laser and autogenous laser processes this paper has demonstrated:

- The clear link between the heat input and the measured distortion for a range of welding processes on $4 \mathrm{~mm}$ thick DH36 ship plate.

- A linear relationship between the fusion area and heat input.

- The advantages and disadvantages of the six welding processes. The comparison suggested that either the hybrid laser or pulsed GMAW were the best processes for this application.

- A simple model which was used to predict the longitudinal residual stress for five of the welds.

- The AWL calculated by the model increased with the heat input primarily due to a widening of the tensile residual stress region. There was a direct link between the AWL predicted by the model and the distortion index, confirming previously published numerical results.

- There is good agreement between the width of the longitudinal residual stresses predicted by the model and those measured experimentally. However, the model fails 
to predict the peak residual stress values correctly which is primarily due to poor yield stress vs. temperature data used in the model.

\section{Acknowledgements}

The authors wish to thank Flemming Nielsen, Brian Brookes, and David Archer from Cranfield University who assisted in the experimental work.

\section{References}

1. K. Masubuchi: 'Analysis of Welded Structures', 642; 1981, Oxford, England, Pergamon Press.

2. D. Radaj: 'Heat Effects of Welding', 1992, New York, Springer.

3. N. A. McPherson: Journal of Ship Production, 2007, 23, 94-117.

4. R. D. James, R. C. Harvey and K. Kyle: 'Guidelines for the Control of Distortion in Thin Ship Structures', Report 42372GDE TDL-98-01, EWI, Columbus, Ohio, 1999.

5. P. Michaleris and A. DeBiccari: Welding Journal, 1997, 76, 172-s-181-s.

6. S. M. Kelly, R. P. Martukanitz, P. Michaleris, M. Bugarewicz, T. D. Huang and L. Kvidahl: Journal of Ship Production, 2006, 22, 105-109.

7. N. A. McPherson, N. Suarez-Fernandez, D. W. Moon, C. P. H. Tan, C. K. Lee and T. N. Baker: Science and Technology of Welding and Joining, 2005, 10, 460-467.

8. D. A. Price, S. W. Williams, A. Wescott, C. J. C. Harrison, A. Rezai, A. Steuwer, M. Peel, P. Staron and M. Kocak: Science and Technology of Welding and Joining, 2007, 12, 620-633.

9. M. Mochizuki, T. Hattori and K. Nakakado: Journal of Engineering Materials and Technology, Transactions of the ASME, 2000, 122, 108-112.

10. A. S. M. Y. Munsi, A. J. Waddell and C. A. Walker: Strain, 2001, 37, 141-149.

11. T. Nagy, S. W. Williams, P. A. Colegrove, I. Fafiolu and C. R. Ikeagu: Proc. 2nd International Workshop on 'Thermal Forming and Welding Distortion', Bremen, Germany, 22-23 April 2008.

12. S. Kou: 'Welding Metallurgy', 461; 2003, New York, USA, Wiley-Interscience.

13. P. W. Fuerschbach: Welding Journal, 1996, 75, 24-s.

14. P. Michaleris, L. Zhang, S. R. Bhide and P. Marugabandhu: Science and Technology of Welding and Joining, 2006, 11, 707-716.

15. P. Dong: Science and Technology of Welding \& Joining, 2005, 10, 389-398.

16. J. Goldak, J. Zhou, S. Tchernov, D. Downey, S. Wang and B. He: Proc. International Conference on 'Trends in Welding Research', Pine Mountain, GA, 16-20 May 2005, Materials Park, OH, USA, 531-539. 
17. D. G. Richards, P. B. Pragnell, P. J. Withers, S. W. Williams, A. Wescott and E. C. Oliver: Materials Science Forum, 2007, 539-543, 4025-4030.

18. R. V. Preston, H. R. Shercliff, P. J. Withers and S. Smith: Acta Materialia, 2004, 52, 4973-4983.

19. N. A. McPherson, K. McGibbon and S. W. Wen: Science and Technology of Welding and Joining, 2006, 11, 191-199.

20. D. Camilleri, T. Comlekci and T. G. F. Gray: Journal of Strain Analysis for Engineering Design, 2005, 40, 161-176.

21. J. Goldak, A. Chakravarti and M. Bibby: Metallurgical Transactions. B, Process Metallurgy, 1984, 15 B, 299-305.

22. W. M. Steen, J. Dowden, M. Davis and P. Kapadia: J. Phys. D, 1988, 21, 1255-1260.

23. J. A. Goldak and M. Akhlaghi: 'Computational Welding Mechanics', 316; 2005, New York, Springer.

24. A. Joseph, D. Harwig, D. F. Farson and R. Richardson: Science and Technology of Welding and Joining, 2003, 8, 400-406. 
Table 1 Composition ranges of DH36 steel.

\begin{tabular}{|c|c|c|c|c|c|c|c|c|c|c|c|c|}
\hline $\mathrm{C}$ & $\mathrm{Mn}$ & $\mathrm{Si}$ & $\mathrm{S}$ & $\mathrm{P}$ & $\mathrm{Al}$ & $\mathrm{Nb}$ & $\mathrm{V}$ & $\mathrm{Cu}$ & $\mathrm{Cr}$ & $\mathrm{Ni}$ & $\mathrm{Mo}$ & $\mathrm{Fe}$ \\
\hline 0.18 & 0.9 & 0.1 & 0.04 & 0.04 & 0.015 & $0.015-$ & $0.05-$ & 0.35 & 0.20 & 0.40 & 0.08 & $\mathrm{Rem}$ \\
$\max$ & -1.6 & -0.5 & $\max$ & $\max$ & $\min$ & 0.05 & 0.1 & & & & & \\
\hline
\end{tabular}

Table 2 Details of welding processes and parameters used.

\begin{tabular}{|c|c|c|c|c|c|c|}
\hline Process & SAW & $\begin{array}{c}\text { DC } \\
\text { GMAW }\end{array}$ & $\begin{array}{l}\text { Pulsed } \\
\text { GMAW }\end{array}$ & CMT & Hybrid Laser & $\begin{array}{c}\text { Autogenous } \\
\text { Laser }\end{array}$ \\
\hline $\begin{array}{l}\text { Machine } \\
\text { Type }\end{array}$ & $\begin{array}{c}\text { Starmatic } \\
\text { 1300DC }\end{array}$ & $\begin{array}{c}\text { ESAB } \\
\text { Aristo } \\
450 / \\
\text { LUD } \\
450\end{array}$ & $\begin{array}{c}\text { ESAB } \\
\text { Aristo } \\
450 / \\
\text { LUD } \\
450\end{array}$ & $\begin{array}{c}\text { Fronius } \\
\text { Transpuls } \\
\text { Synergic } \\
\text { 5000 CMT } \\
\text { power supply } \\
\text { with VR700 } \\
\text { wire CMT } \\
\text { feeder }\end{array}$ & $\begin{array}{c}\text { IPG YLR-8000 } \\
\text { fibre laser with } \\
\text { Lincoln Power } \\
\text { Wave } \\
\text { 455M/STT } \\
\text { operating in } \\
\text { pulsed mode. }\end{array}$ & $\begin{array}{c}\text { IPG YLR- } \\
8000 \text { fibre } \\
\text { laser }\end{array}$ \\
\hline $\begin{array}{l}\text { Contact } \\
\text { tip to } \\
\text { work } \\
\text { distance } \\
\text { (mm) }\end{array}$ & 20 & 12 & 12 & 12 & $\begin{array}{l}20,69^{\circ} \text { to laser } \\
\text { torch. }\end{array}$ & - \\
\hline $\begin{array}{l}\text { Welding } \\
\text { speed } \\
\left(\mathrm{m} \mathrm{min}^{-1}\right)\end{array}$ & 1.125 & 0.667 & 0.8 & 0.618 & 2.5 & 2.5 \\
\hline $\begin{array}{l}\text { WFS } \\
\left(\mathrm{m} \mathrm{min}^{-1}\right)\end{array}$ & 1.4 & 7.6 & 8.5 & 6.9 & 11.43 & - \\
\hline $\begin{array}{l}\text { Average } \\
\text { Current } \\
\text { (A) }\end{array}$ & 503.5 & 278 & 281 & 232 & 270 & - \\
\hline $\begin{array}{l}\text { Average } \\
\text { Voltage } \\
\text { (V) }\end{array}$ & 35 & 25.5 & 27.5 & 16.5 & 25 & - \\
\hline $\begin{array}{l}\text { Gas Flow } \\
\left(\mathrm{L} \mathrm{min}^{-1}\right)\end{array}$ & - & 15 & 15 & 15 & 12 & 12 \\
\hline $\begin{array}{l}\text { Laser } \\
\text { power } \\
(\mathrm{kW}) \\
\end{array}$ & - & - & - & - & 4.5 & 4.5 \\
\hline $\begin{array}{l}\text { Laser } \\
\text { spot size } \\
(\mathrm{mm})\end{array}$ & - & - & - & - & $\begin{array}{c}0.63 \\
\text { (uniform) }\end{array}$ & $\begin{array}{c}0.63 \\
\text { (uniform) }\end{array}$ \\
\hline $\begin{array}{l}\text { Focus } \\
\text { position }\end{array}$ & & & & & $\begin{array}{l}\text { On top surface. } \\
\text { Angle between } \\
\text { the torch and } \\
\text { the laser was } \\
\text { set at } 31^{\circ} .\end{array}$ & $\begin{array}{l}\text { On top } \\
\text { surface }\end{array}$ \\
\hline
\end{tabular}


Table 3 Subjective comparison of the six welding processes.

\begin{tabular}{|c|c|c|c|c|c|c|c|c|c|}
\hline Process & Edge Prep & Welding Position & Safety & Cost & Productivity & $\begin{array}{l}\text { Mechanical } \\
\text { Properties }\end{array}$ & Quality & Heat Input & Distortion \\
\hline SAW & None & Down hand & Standard & Medium & Medium & Good & Good & Very high & Very High \\
\hline DC GMAW & None & Down hand & Standard & Low & Medium & Good & Poor & High & High \\
\hline Pulsed GMAW & None & Any & Standard & Low & Medium & Good & Good & High & Medium \\
\hline CMT & V- Butt & Any & Standard & Medium & Low to medium & Good & Good & Medium & Medium \\
\hline Hybrid Laser & None & Any & Very high & Very High & High & Good & Good & Low & Low \\
\hline Autogenous Laser & None & Any & Very high & High & High & Poor & Poor & Low & Low \\
\hline
\end{tabular}

Key

$\square$ Good $\quad \square$ Fair $\quad \square$ Poor 
Table 4 Size parameters used in heat source model.

\begin{tabular}{|c|c|c|c|}
\hline Process & $\begin{array}{c}\mathrm{a}(\mathrm{mm}) \\
\text { (Half width of weld } \\
\text { pool) }\end{array}$ & $\begin{array}{c}\mathrm{c}_{\mathrm{f}}(\mathrm{mm}) \\
\text { (Size of weld pool in } \\
\text { front of arc) }\end{array}$ & $\begin{array}{c}\mathrm{c}_{\mathrm{b}}(\mathrm{mm}) \\
\text { (Size of weld pool } \\
\text { behind arc) }\end{array}$ \\
\hline GMAW & 6 & 9 & 18 \\
\hline Pulsed GMAW & 6 & 6 & 21 \\
\hline CMT & 5 & 7 & 14 \\
\hline Hybrid Laser & 3.5 & 5 & 15 \\
\hline $\begin{array}{c}\text { Autogenous } \\
\text { Laser }\end{array}$ & 1 & 1 & 1 \\
\hline
\end{tabular}

Table 5 Assumed room temperature yield strength for the different welding processes.

\begin{tabular}{|c|c|c|c|}
\hline $\begin{array}{c}\text { Welding } \\
\text { Process }\end{array}$ & $\begin{array}{c}\text { Average Weld Metal } \\
\text { Hardness (HV 0.2) }\end{array}$ & $\begin{array}{c}\text { Approximate Tensile } \\
\text { Strength (MPa) }\end{array}$ & $\begin{array}{c}\text { Approximate Yield } \\
\text { Strength (MPa) }\end{array}$ \\
\hline GMAW & 224 & 715 & 451 \\
\hline Pulsed & 234 & 785 & 495 \\
GMAW & 226 & 738 & 465 \\
\hline CMT & 258 & 820 & 517 \\
\hline Hybrid Laser & 414 & 1390 & 876 \\
\hline $\begin{array}{c}\text { Autogenous } \\
\text { Laser }\end{array}$ & & & \\
\hline
\end{tabular}

Table 6 Weld efficiency and convective heat transfer coefficients used in numerical model.

\begin{tabular}{|c|c|c|}
\hline Welding Process & Weld Efficiency $(\boldsymbol{\%})$ & $\begin{array}{c}\text { Convective Heat Transfer Coefficient (W } \\
\left.\mathbf{m}^{-\mathbf{2}} \mathbf{K}^{\mathbf{- 1}}\right)\end{array}$ \\
\hline GMAW & 70 & 100 \\
\hline Pulsed GMAW & 58 & 20 \\
\hline CMT & 90 & 20 \\
\hline Hybrid Laser & 72 & 90 \\
\hline Autogenous Laser & 70 & 80 \\
\hline
\end{tabular}



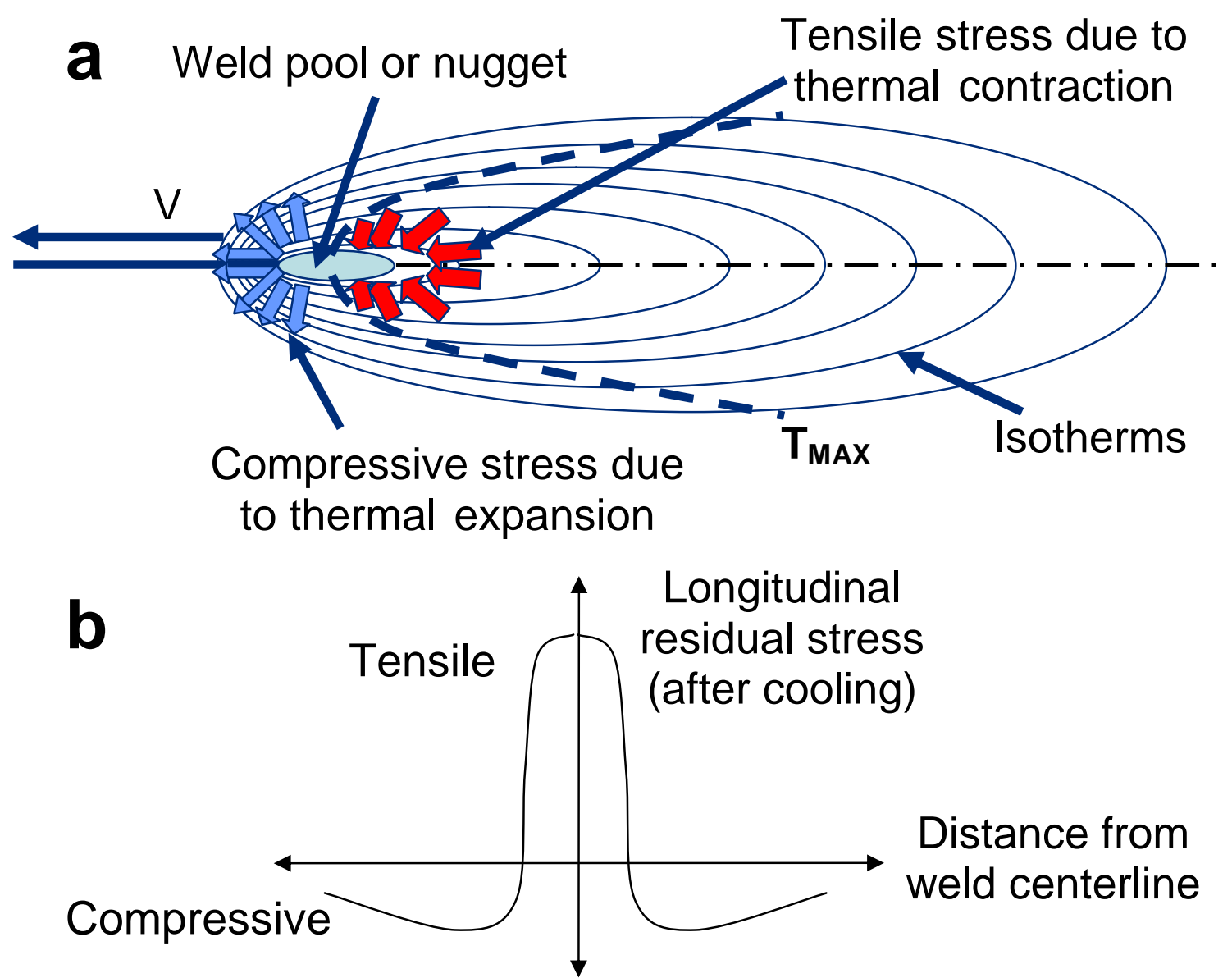

Fig. 1 Stresses near a typical weld: (a) thermal stresses during and (b) longitudinal residual stresses after welding.

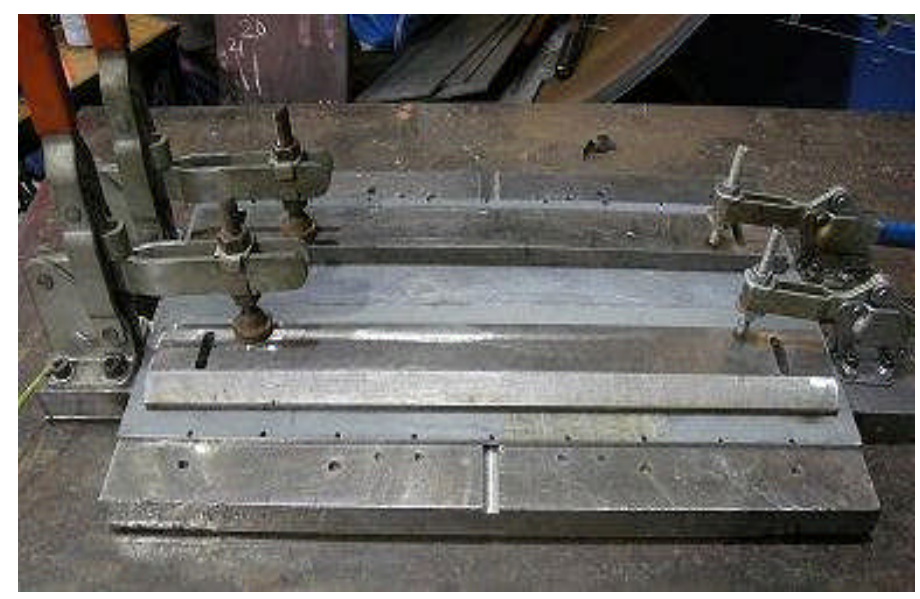

Fig. 2 Clamping arrangement for plates. 


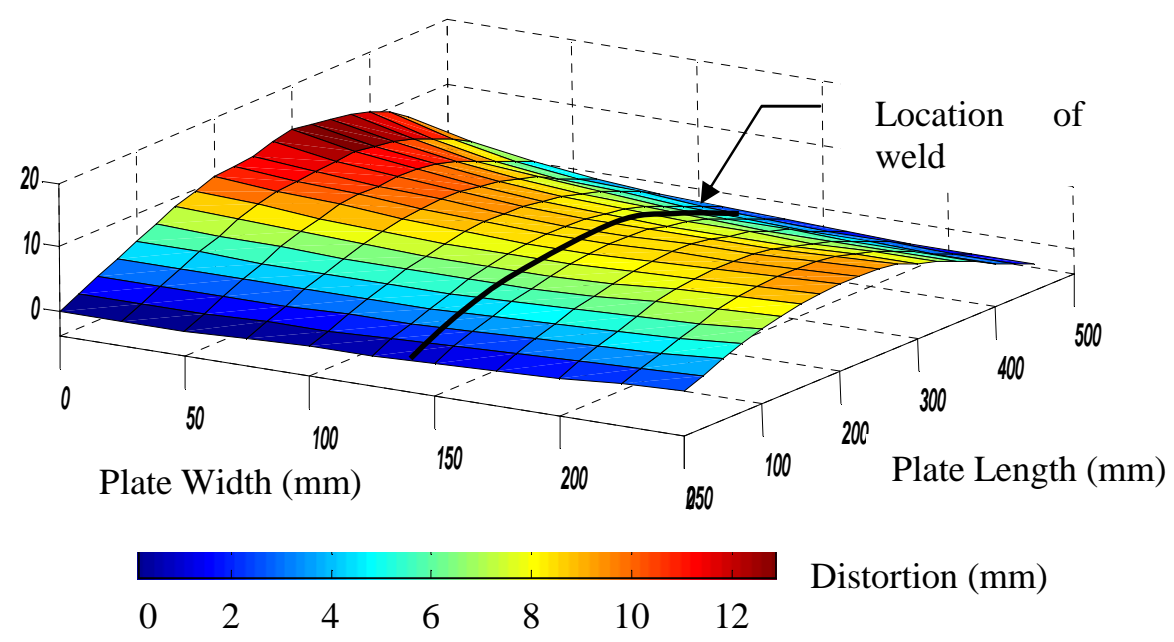

Fig. 3 Measured distortion profile for SAW
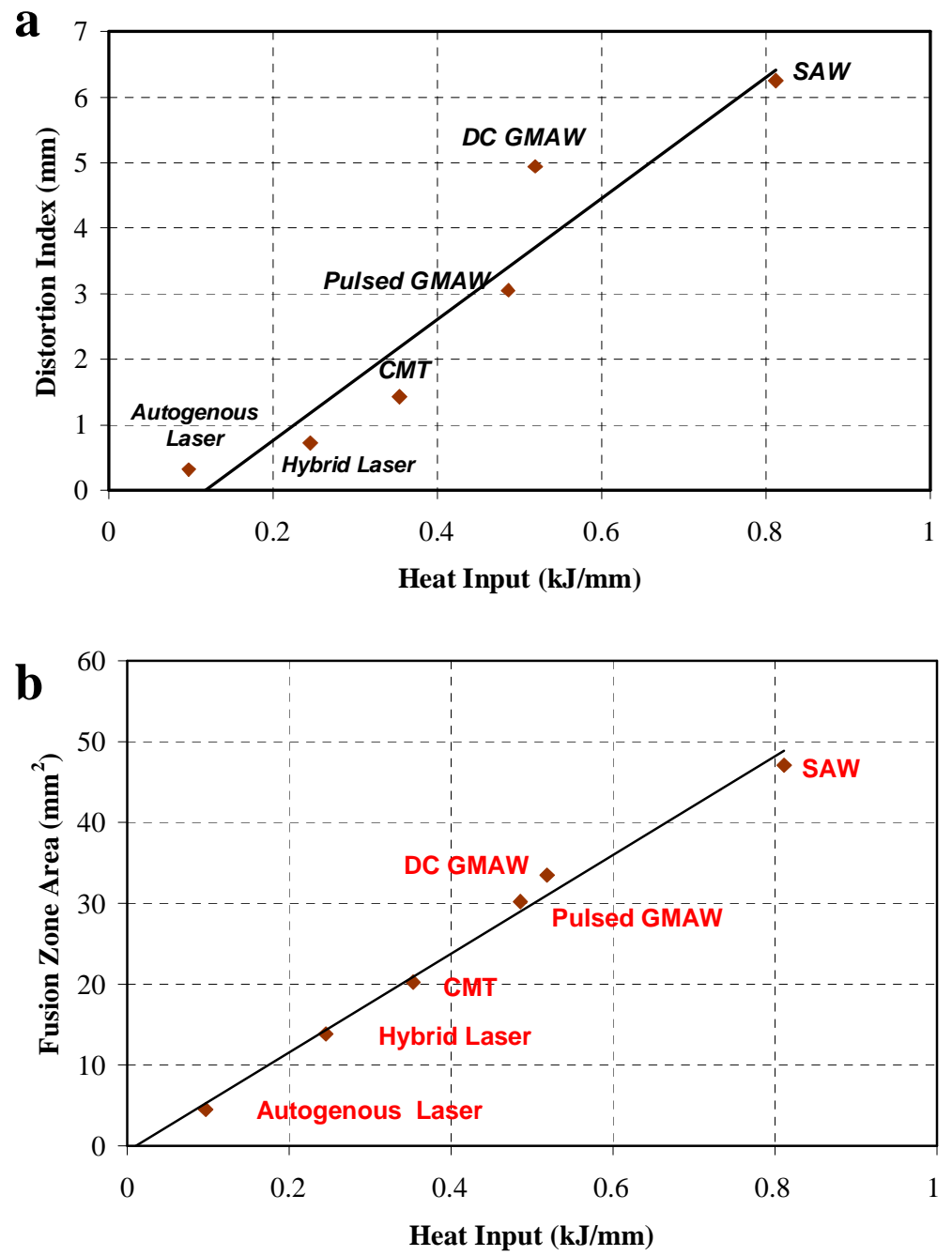

Fig. 4 (a) Distortion index (trend line for textbook efficiencies) and (b) fusion zone area vs. the heat input. 


\section{$\mathbf{a}$}

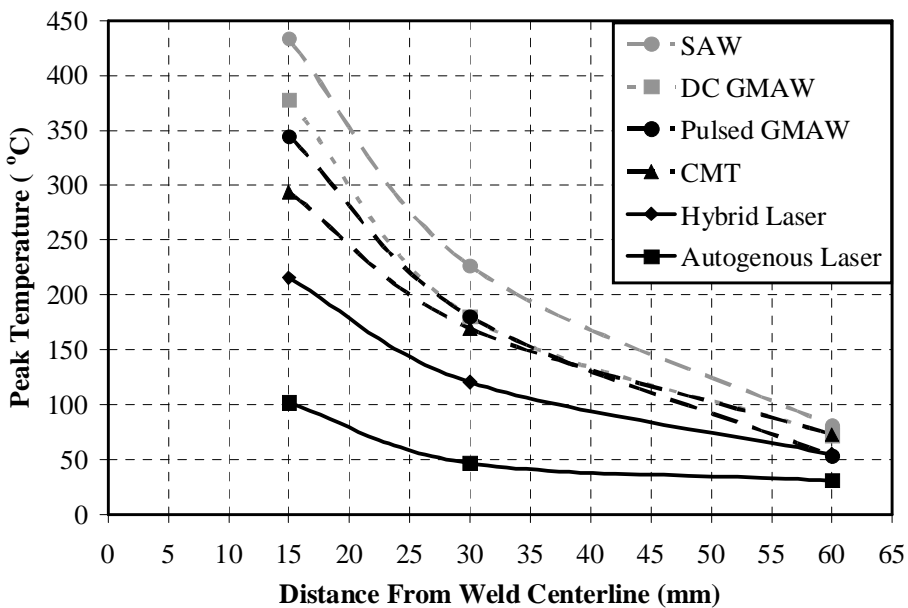

b

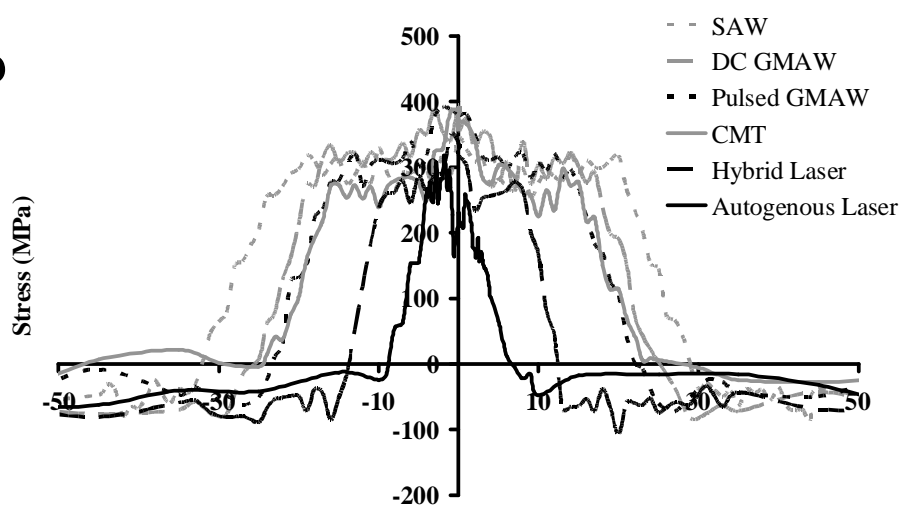

Distance from Weld Centreline (mm)

c

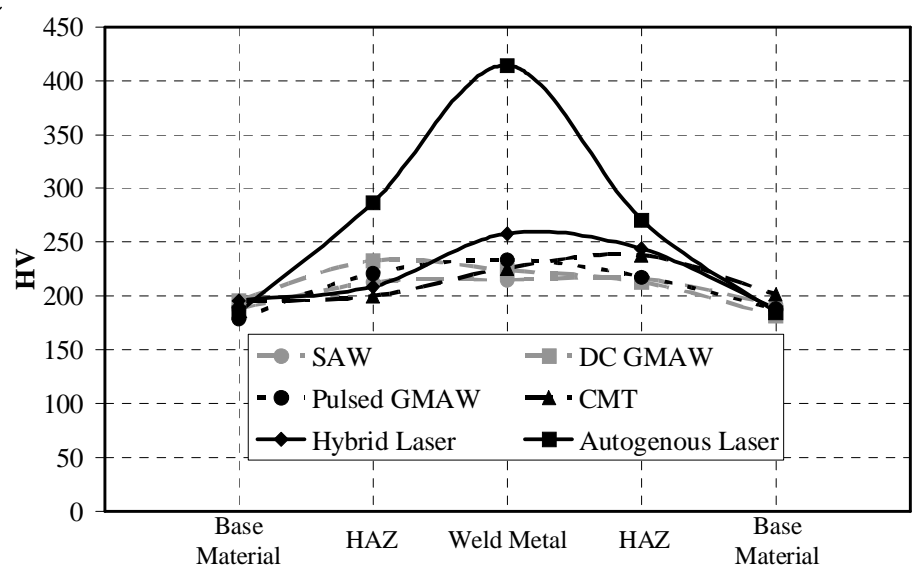

Fig. 5 Comparison of (a) peak temperature, (b) longitudinal residual stress, and (c) hardness between the different welding processes. 

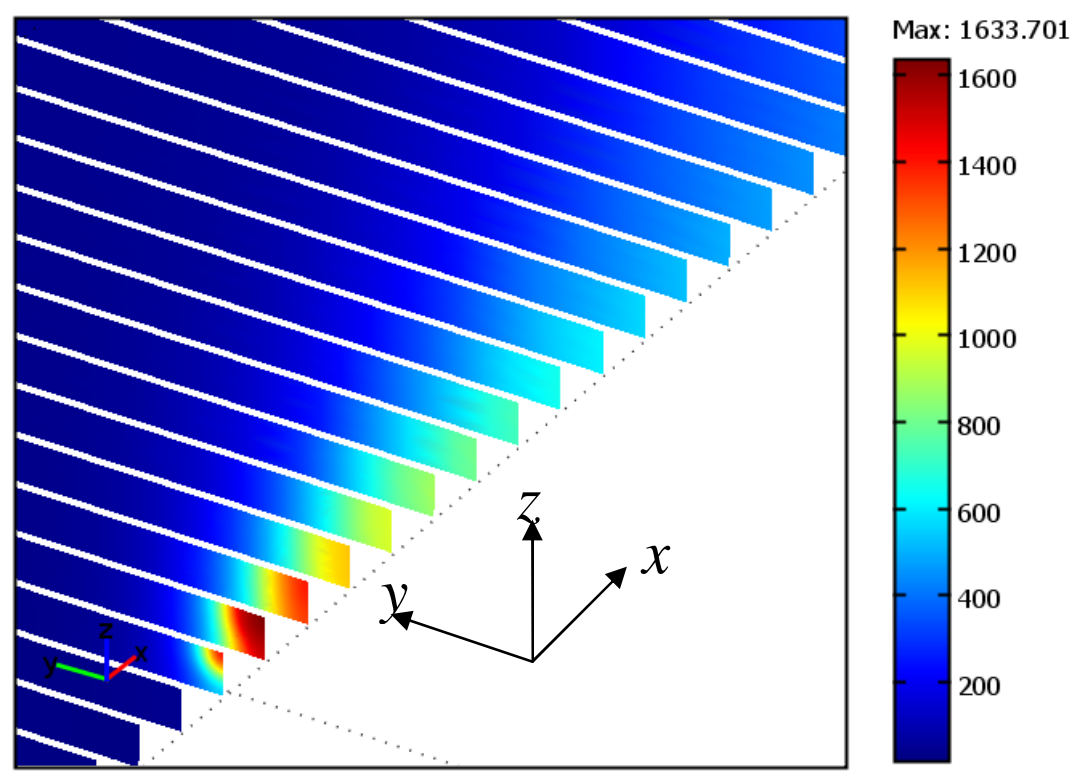

Min: 19.24

Fig. 6 Division of thermal solution for sequential stress analysis.

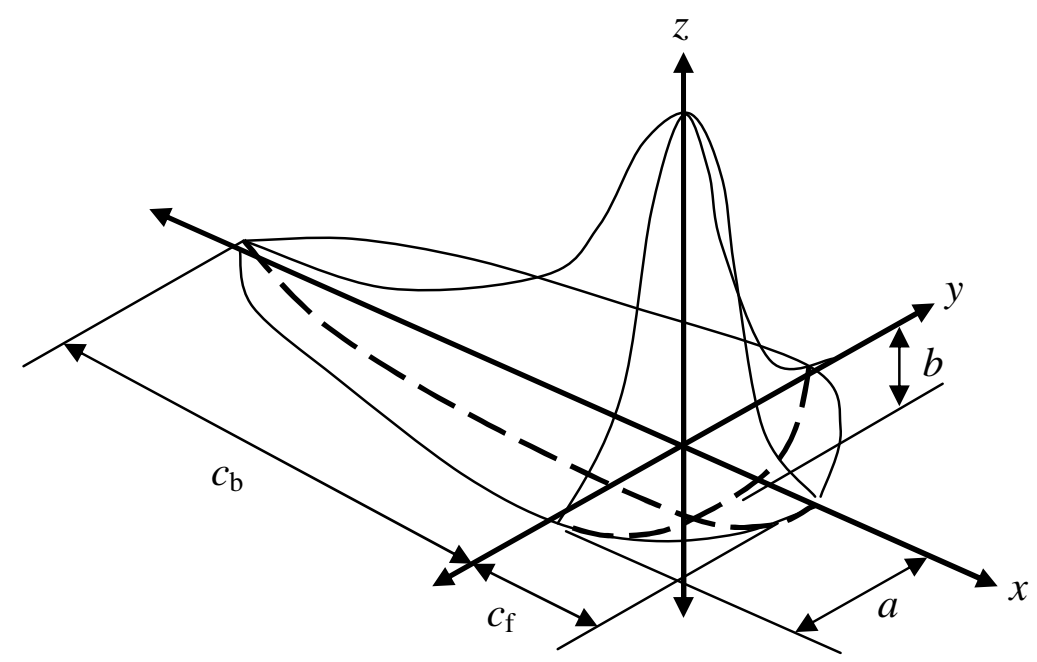

Fig. 7 Definition of coordinate system and coefficients used for heat source.

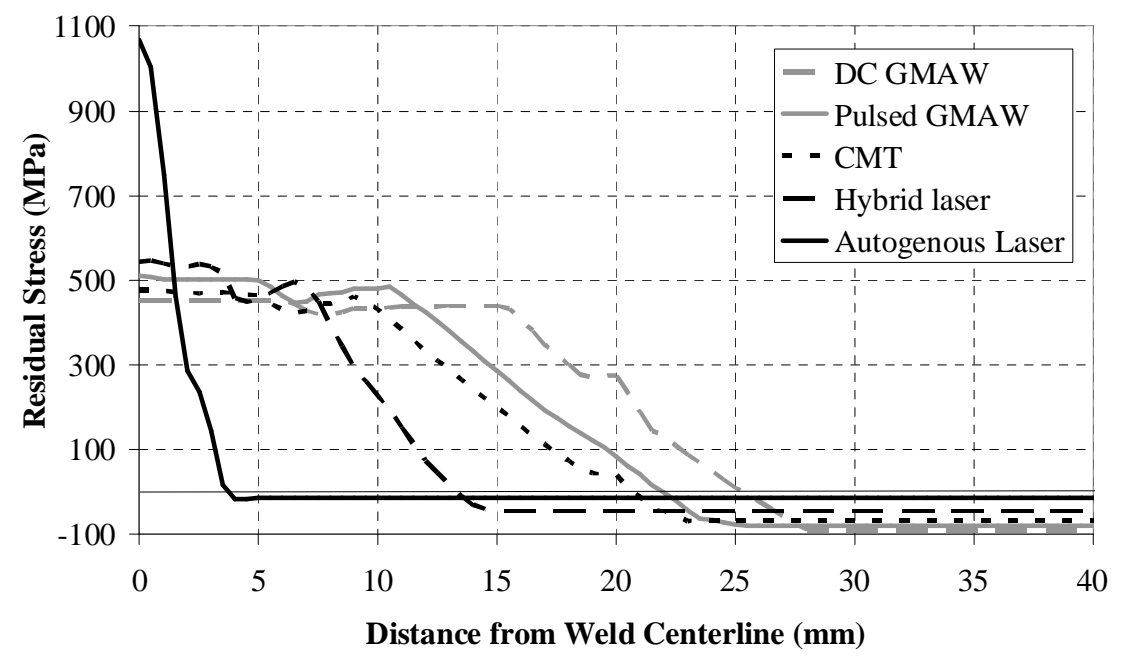

Fig. 8 Predicted longitudinal residual stress. 


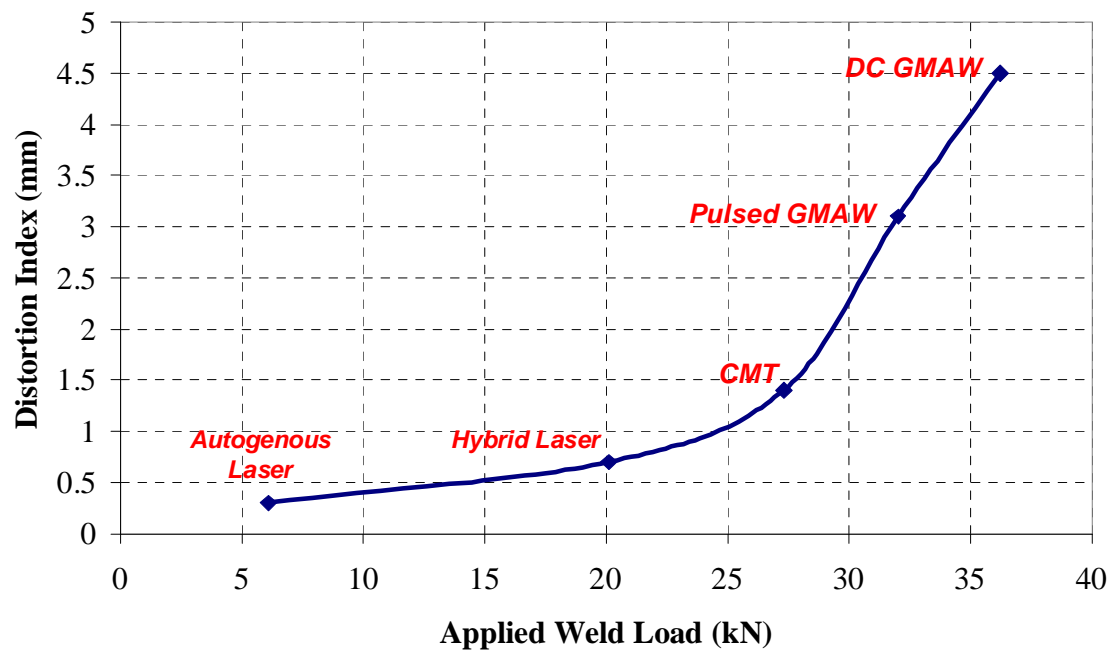

Fig. 9 Correlation between the distortion index and the AWL. 

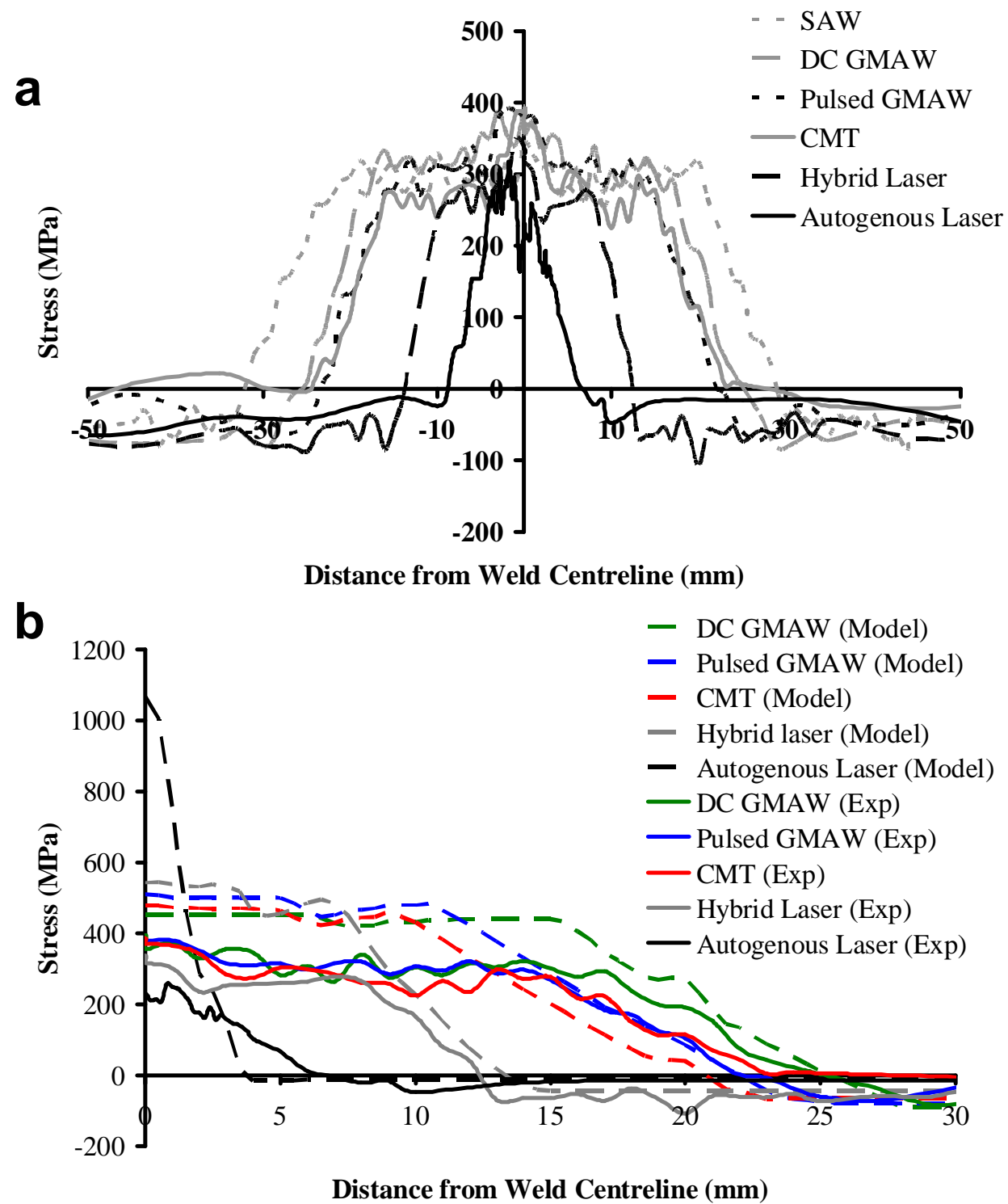

Fig. 10 (a) Longitudinal residual stress measured across the six samples and (b) comparison with the model predictions. 